\title{
The OPE of the B-meson light-cone wavefunction for exclusive $B$ decays: radiative corrections and higher-dimensional operators
}

\author{
Hiroyuki Kawamura \\ Radiation Laboratory, RIKEN, Wako 351-0198, Japan \\ E-mail: hiroyukieribf.riken.jp

\section{Jiro Kodaira*} \\ Theory Division, KEK, Tsukuba 305-0801, Japan

\section{Kazuhiro Tanaka ${ }^{\dagger}$} \\ Department of Physics, Juntendo University, Inba, Chiba 270-1695, Japan \\ E-mail: tanakakesakura.juntendo.ac.jp
}

\begin{abstract}
We discuss the $B$-meson light-cone wavefunction relevant for QCD factorization approach for exclusive $B$-meson decays. We derive the operator product expansion for the $B$-meson light-cone wavefunction, taking into account the local composite operators of dimension less than 6 and calculating the radiative corrections at order $\alpha_{s}$ for the corresponding Wilson coefficients. The result embodies peculiar UV and IR behaviors of the $B$-meson light-cone wavefunction, the Sudakovtype double logarithmic effects and the mixing of the multiparticle states with additional gluons inside the $B$ meson. The former effects are induced from the cusp singularity in the radiative corrections, while the latter is manifested by the participation of the higher-dimensional operators associated with the nonperturbative structure of the $B$ meson.
\end{abstract}

8th International Symposium on Radiative Corrections

October 1-5, 2007

Florence, Italy

* Deceased.

† Speaker. 
For the exclusive $B$-meson decays such as $B \rightarrow \pi \pi, \rho \gamma, \ldots$, systematic methods have been developed using QCD factorization based on the heavy-quark limit [1]. Among the building blocks in the corresponding factorization formula of the decay amplitude, essential roles are played by the light-cone wavefunctions (LCWFs) for the participating mesons, which include nonperturbative long-distance contribution. In particular, not only the LCWFs for the light mesons $\pi, \rho, \ldots$, produced in the final state, but also those for the $B$ meson participate in processes where large momentum is transferred to the soft spectator quark via hard gluon exchange [1]. We define the leading quark-antiquark component of $B$-meson LCWF as the vacuum-to-meson matrix element [2]:

$$
\tilde{\phi}_{B}(t, \mu)=\left\langle 0\left|\bar{q}(t n) \mathrm{P} e^{i g \int_{0}^{t} d \lambda n \cdot A(\lambda n)} \not h \gamma_{5} h_{v}(0)\right| \bar{B}(v)\right\rangle=\int d \omega e^{-i \omega t} \phi_{B}(\omega, \mu) .
$$

Here the bilocal operator is built of the $b$-quark and light antiquark fields, $h_{v}(0)$ and $\bar{q}(t n)$, linked by the Wilson line at a light-like separation $t n$ with $n_{\mu}$ the light-like vector $\left(n^{2}=0, n \cdot v=1\right), v_{\mu}$ is 4-velocity of the $B$ meson, and the RHS defines the momentum representation with $\omega v^{+}$denoting the LC component of the light antiquark's momentum. A difference compared with the familiar pion-LCWF is that $h_{v}(0)$ is an effective field in the heavy-quark effective theory (HQET) [3]. In (1), $\mu$ denotes the scale where the bilocal operator is renormalized. If one could Taylor expand the bilocal operator, the WF (1) would be equivalent to matrix element of a set of local operators of the type, $\bar{q}(0)(n \cdot D)^{j} h h \gamma_{5} h_{v}(0)$. Indeed, for the light mesons $\pi, \rho, \ldots$, such correspondence holds and is useful for establishing systematic framework to calculate the LCWFs explicitly [4]. In contrast, for the $B$-meson LCWF (1), such straightforward correspondence is broken as demonstrated below, because the twist is not a good quantum number by presence of the effective heavy-quark field.

The "IR structure" of the $B$-meson LCWF has been studied [5] using constraints from equations of motion (EOM), $\bar{q} \overleftarrow{I D}=v \cdot \vec{D} h_{v}=0$, and heavy-quark symmetry, $v h_{v}=h_{v}$. These constraints are solved to give (1) as the sum of the two terms, $\phi_{B}(\omega)=\phi_{B}^{(W W)}(\omega)+\phi_{B}^{(g)}(\omega)$. The first term is expressed by the analytic formula as $\phi_{B}^{(W W)}(\omega)=i F \omega \theta(2 \bar{\Lambda}-\omega) /\left(2 \bar{\Lambda}^{2}\right)$, in terms of the HQET parameters [3], the decay constant $F=-i\left\langle 0\left|\bar{q} h \gamma_{5} h_{v}\right| \bar{B}(v)\right\rangle$ and the "effective mass" $\bar{\Lambda}=m_{B}-m_{b}$ representing the mass difference between $B$-meson and $b$-quark. The second term $\phi_{B}^{(g)}$ is obtained as a certain integral of matrix element of 3-body LC operator, $\left\langle 0\left|\bar{q}(t n) G_{\alpha \beta}(u n) \sigma_{\lambda \eta} h_{v}(0)\right| \bar{B}(v)\right\rangle$, where ${ }^{1}$ the nonperturbative gluons generated inside the $B$ meson participate as the field strength tensor $G_{\alpha \beta}$. On the other hand, the "UV structure" of the $B$-meson WF has been studied in [6] by calculating the 1-loop renormalization of the bilocal operator in (1). The "vertex-type" correction around a "cusp" between the two Wilson lines, the light-like one of (1) and the time-like one from $h_{v}(0)=\operatorname{P} \exp \left[i g \int_{-\infty}^{0} d \lambda v \cdot A(\lambda v)\right] h_{v}(-\infty v)$, produces the "radiation tail" as $\phi_{B}(\omega, \mu) \sim$ $-i F C_{F} \alpha_{s} \ln (\omega / \mu) /(\pi \omega)$ for $\omega \gg \mu$, where $C_{F}=\left(N_{c}^{2}-1\right) /\left(2 N_{c}\right)$. This implies that the moments $\int_{0}^{\infty} d \omega \omega^{j} \phi_{B}(\omega, \mu)$, which would correspond to matrix element of the local operators mentioned above, are divergent reflecting the singularity associated with the cusp of the two Wilson lines [6].

To see the behavior of the $B$-meson WF incorporating both IR and UV structures, we calculate the radiative corrections taking into account hard and soft/collinear loops. We calculate the 1-loop diagrams for the 2-point function (1) in $4-2 \varepsilon$ dimensions. We first note that the similar diagrams for the case of the pion LCWF, defined by the light- $q \bar{q}$ bilocal operator, yield the "cancelling" UV

\footnotetext{
${ }^{1}$ The Wilson lines connecting the constituent fields are suppressed for simplicity.
} 
and IR poles, $1 / \varepsilon_{U V}-1 / \varepsilon_{I R}$, reflecting the scaleless loop-integral in massless $\mathrm{QCD}$, and this structure is accompanied by the convolution with the splitting function (Brodsky-Lepage kernel) [4]; the corrections are analytic (Taylor expandable) at $t=0$. Now, for the $B$-meson LCWF (1), we get $^{1}$

$$
\begin{array}{r}
\tilde{\phi}_{B}^{1 \text {-loop }}(t, \mu)=\frac{\alpha_{S} C_{F}}{2 \pi} \int_{0}^{1} d \xi\left[\left\{-\left(\frac{1}{2 \varepsilon_{U V}^{2}}+\frac{L}{\varepsilon_{U V}}+L^{2}+\frac{5 \pi^{2}}{24}\right) \delta(1-\xi)+\left(\frac{1}{\varepsilon_{U V}}-\frac{1}{\varepsilon_{I R}}\right)\left[\frac{\xi}{1-\xi}\right]_{+}\right.\right. \\
\left.\left.-\left(\frac{1}{2 \varepsilon_{I R}}+L\right)\right\}\left\langle\bar{q}(\xi \text { tn }) h \gamma_{5} h_{v}(0)\right\rangle-t\left(\frac{1}{\varepsilon_{I R}}+2 L-1-\xi\right)\left\langle\bar{q}(\xi \text { tn }) v \cdot \overleftarrow{D} \not h \gamma_{5} h_{v}(0)\right\rangle\right]+\cdots, \text { (2) }
\end{array}
$$

where $L \equiv \ln \left(i t \mu e^{\gamma_{E}}\right)$ with the $\overline{\mathrm{MS}}$ scale $\mu$, and $\langle\cdots\rangle \equiv\langle 0|\cdots| \bar{B}(v)\rangle$. The "vertex-type" correction that connects the light-like Wilson line and the $\bar{q}(t n)$ field in (1) is associated with only the massless degrees of freedom, and gives the term with the "plus"-distribution $[\xi /(1-\xi)]_{+}$, which has the same structure as the corresponding correction in the pion case. But the other diagrams give novel behavior with "non-cancelling" UV and IR poles: the another vertex-type correction gives [6] the terms proportional to $\delta(1-\xi)$, which contain the double as well as single UV pole, corresponding to the cusp singularity mentioned above. The "ladder-type" correction, connecting the two quark fields in (1), gives the last line in (2), which contains the IR poles and is associated with not only the bilocal operator in (1) but also the higher dimensional operators; note that the ellipses in (2) are expressed by those operators involving the two or more additional covariant derivatives.

The renormalized LCWF, obtained by subtracting the UV poles from (2), is non-analytic at $t=0$ by presence of logarithms $L, L^{2}$ [6, 7]. In particular, the nontrivial dependence on $t \mu$ through $L$ implies that the scale $\sim 1 / t$ plays a role to separate the UV and IR regions. Thus we have to use the operator product expansion (OPE) to treat the different UV and IR behaviors simultaneously: the coefficient functions absorb all the singular logarithms, while, for the local operators to absorb the IR poles, we have to take into account many higher dimensional operators. Such OPE with local operators is useful when the separation $t$ is less than the typical distance scale of quantum fluctuation, i.e., when $t \lesssim 1 / \mu$. The OPE result can be evolved to a higher scale by the evolution operator associated with the single-UV-pole terms in (2), i.e., by the Brodsky-Lepage-type kernel and the Sudakov-type operator with the cusp anomalous dimension [6, 7].

An OPE for the $B$-meson LCWF (1) has been discussed in [8], taking into account the local operators of dimension less than 5 and the $\mathrm{NLO}\left(O\left(\alpha_{s}\right)\right)$ corrections to the corresponding Wilson coefficients in a "cutoff scheme", where specifically an additional momentum cutoff $\Lambda_{U V}$ is introduced and the OPE is derived for the regularized moments, $\int_{0}^{\Lambda_{U V}} d \omega \omega^{j} \phi_{B}(\omega, \mu)$ with $j=0,1$. In this work we derive the OPE for the $B$-meson LCWF (1), taking into account the local operators of dimension less than 6 and calculating the radiative corrections for the corresponding Wilson coefficients at NLO accuracy. Following the above discussion, we carry out the calculation in the $\overline{\mathrm{MS}}$ scheme for $t \lesssim 1 / \mu$, so that there is no need to introduce any additional cutoff.

The most complicated part of this calculation is to reorganize the contributions from a gaugeinvariant set of Feynman diagrams in terms of matrix element of gauge-invariant operators including higher dimensional ones. In particular, to derive the Wilson coefficients associated with the dimension-5 operators such as $\bar{q} G_{\alpha \beta} \not h \gamma_{5} h_{v}$, we have to compute the 1-loop diagrams for the 3-point function, as well as those for the 2-point function as in (2), where the former diagrams are obtained by attaching the external gluon line to the latter diagrams in all possible ways. To perform the calculation in a systematic and economic way, we employ the background field method [9]. We de- 
compose the quark and gluon fields into the "quantum" and "classical" parts, where the latter part represents the nonperturbative long-distance degrees of freedom inside $B$ meson as a background field and satisfies the classical EOM. The quark and gluon propagators for the quantum part contain the coupling with an arbitrary number of background fields, and each building block of the Feynman diagrams obeys the exact transformation property under the gauge transformation for the background fields. We use the Fock-Schwinger gauge, $x^{\mu} A_{\mu}^{(c)}(x)=0$, for the background gluon field $A_{\mu}^{(c)}$. This gauge condition is solved to give $A_{\mu}^{(c)}(x)=\int_{0}^{1} d u u x^{\beta} G_{\beta \mu}^{(c)}(u x)$ [9], which allows us to reexpress each Feynman diagram in terms of matrix element of the operators associated with the field strength tensor.

Using this framework, the tree-level matching to derive our OPE can be performed replacing each constituent field in (1) by the corresponding background fields. The classical bilocal operator can be Taylor expanded, and we obtain the OPE at the tree level with the local operators of dimension-3, -4, and -5 (see (3) below).

For the 1-loop matching, we calculate the 1-loop corrections to the 2- and 3-point functions with the insertion of the bilocal operator in (1), taking into account the mixing of operators of dimension less than 6. Apparently the mixing through the UV region of the loop momenta arises only in the 2-point function, whose result can be immediately obtained from (2); but the additional mixing can arise in both 2- and 3-point functions accompanying the IR poles. We perform the loop integration in the coordinate space using the Schwinger ("heat kernel") representation of the Feynman amplitudes under the background fields [9]. For the calculation of the 3-point function, the Fock-Schwinger gauge ensures that the Wilson line in (1), as well as the heavy-quark field, does not couple directly to the background gluons. We reorganize the result in terms of a complete set of gauge-invariant operators using the EOM and heavy-quark symmetry. We subtract the UV poles to renormalize the bilocal operator of (1), and also renormalize the local operators to absorb the IR poles. Combining this result with the above tree-level result, we obtain the OPE for the bilocal operator in (1), $\bar{q}(t n) \mathrm{P} e^{i g \int_{0}^{t} d \lambda n \cdot A(\lambda n)} n \gamma_{5} h_{v}(0)=\sum_{i} C_{i}(t, \mu) \mathscr{O}_{i}(\mu)$, to the desired accuracy as

$$
\begin{gathered}
\bar{q}(t n) \mathrm{P} e^{i g \int_{0}^{t} d \lambda n \cdot A(\lambda n)} n h \gamma_{5} h_{v}(0)=\left[1-\frac{\alpha_{s} C_{F}}{4 \pi}\left(2 L^{2}+2 L+\frac{5 \pi^{2}}{12}\right)\right] \mathscr{O}_{1}^{(3)} \\
-i t\left\{\left[1-\frac{\alpha_{s} C_{F}}{4 \pi}\left(2 L^{2}+L+\frac{5 \pi^{2}}{12}\right)\right] \mathscr{O}_{1}^{(4)}-\frac{\alpha_{s} C_{F}}{4 \pi}(4 L-3) \mathscr{O}_{2}^{(4)}\right\} \\
-\frac{t^{2}}{2}\left\{\left[1-\frac{\alpha_{s} C_{F}}{4 \pi}\left(2 L^{2}+\frac{2}{3} L+\frac{5 \pi^{2}}{12}\right)\right] \mathscr{O}_{1}^{(5)}-\frac{\alpha_{s} C_{F}}{4 \pi}\left(4 L-\frac{10}{3}\right)\left(\mathscr{O}_{2}^{(5)}+\mathscr{O}_{3}^{(5)}\right)\right. \\
+\frac{\alpha_{s}}{4 \pi}\left[C_{F}\left(-4 L+\frac{10}{3}\right)+C_{G}\left(7 L-\frac{13}{2}\right)\right] \mathscr{O}_{4}^{(5)}+\frac{\alpha_{s}}{4 \pi}\left(-\frac{4}{3} C_{F}+C_{G}\right)(L-1) \mathscr{O}_{5}^{(5)} \\
\left.+\frac{\alpha_{s}}{4 \pi}\left(-\frac{2}{3} C_{F}+C_{G}\right)(L-1) \mathscr{O}_{6}^{(5)}+\frac{\alpha_{s}}{4 \pi}\left(-\frac{1}{3} C_{F}+\frac{1}{4} C_{G}\right)(L-1) \mathscr{O}_{7}^{(5)}\right\}
\end{gathered}
$$

where $C_{G}=N_{c}$ and a basis of local operators, $\mathscr{O}_{k}^{(d)}(k=1,2, \ldots)$, of dimension- $d$ is defined as $\mathscr{O}_{1}^{(3)} \equiv \bar{q} h \gamma_{5} h_{v},\left\{\mathscr{O}_{k}^{(4)}\right\} \equiv\left\{\bar{q}(i n \cdot \overleftarrow{D}) h \gamma_{5} h_{v}, \bar{q}(i v \cdot \overleftarrow{D}) h \gamma_{5} h_{v}\right\}$, and $\left\{\mathscr{O}_{k}^{(5)}\right\} \equiv\left\{\bar{q}(i n \cdot \overleftarrow{D})^{2} \not h \gamma_{5} h_{v}\right.$, $\bar{q}(i v \cdot \overleftarrow{D})(i n \cdot \overleftarrow{D}) \not h \gamma_{5} h_{v}, \bar{q}(i v \cdot \overleftarrow{D})^{2} \not h \gamma_{5} h_{v}, \bar{q} i g G_{\alpha \beta} v^{\alpha} n^{\beta} \not h \gamma_{5} h_{v}, \bar{q} i g G_{\alpha \beta} \gamma^{\alpha} n^{\beta} \not{h} \gamma_{5} h_{v}, \bar{q} i g G_{\alpha \beta} \gamma^{\alpha} v^{\beta} \not{h} \gamma_{5} h_{v}$, $\left.\bar{q} g G_{\alpha \beta} \sigma^{\alpha \beta} \not h \gamma_{5} h_{v}\right\}$. Here we have introduced another light-like vector, $\bar{n}^{2}=0$, as $v_{\mu}=\left(n_{\mu}+\bar{n}_{\mu}\right) / 2$. The double $\operatorname{logarithm} L^{2}$ in the coefficient functions originates from cusp singularity. The 1-loop 
corrections for the 3-point function induce only the operators $\mathscr{O}_{4,5,6,7}^{(5)}$ associated with the field strength tensor, while those for the 2-point function induce all ten operators through the use of the EOM.

Taking the matrix element $\langle\cdots\rangle \equiv\langle 0|\cdots| \bar{B}(v)\rangle$ of (3), we can derive the OPE form of the $B$ meson LCWF (1). Matrix elements of the local operators appearing in the RHS of (3) are known to be related to a few nonperturbative parameters in the HQET, using the EOM and heavy-quark symmetry as demonstrated in [2, 5]: $\left\langle\mathscr{O}_{1}^{(4)}\right\rangle=4 i F \bar{\Lambda} / 3,\left\langle\mathscr{O}_{2}^{(4)}\right\rangle=i F \bar{\Lambda}$, where $F$ and $\bar{\Lambda}$ were introduced below (1), and all seven matrix elements $\left\langle\mathscr{O}_{k}^{(5)}\right\rangle$ for the dimension-5 operators can be expressed by $F, \bar{\Lambda}$ and two additional HQET parameters $\lambda_{E}$ and $\lambda_{H}$, which are associated with the chromoelectric and chromomagnetic fields inside the $B$ meson, respectively, as $\left\langle\bar{q} g \mathbf{E} \cdot \alpha \gamma_{5} h_{v}\right\rangle=F \lambda_{E}^{2}$ and $\left\langle\bar{q} g \mathbf{H} \cdot \sigma \gamma_{5} h_{v}\right\rangle=i F \lambda_{H}^{2}$ in the rest frame, $v=(1, \mathbf{0})$. We get the OPE form for the LCWF (1) as

$$
\begin{gathered}
\tilde{\phi}_{B}(t, \mu)=i F(\mu)\left\{1-\frac{\alpha_{s} C_{F}}{4 \pi}\left(2 L^{2}+2 L+\frac{5 \pi^{2}}{12}\right)-i t \frac{4 \bar{\Lambda}}{3}\left[1-\frac{\alpha_{s} C_{F}}{4 \pi}\left(2 L^{2}+4 L-\frac{9}{4}+\frac{5 \pi^{2}}{12}\right)\right]\right. \\
-t^{2} \bar{\Lambda}^{2}\left[1-\frac{\alpha_{s} C_{F}}{4 \pi}\left(2 L^{2}+\frac{16}{3} L-\frac{35}{9}+\frac{5 \pi^{2}}{12}\right)\right]-\frac{t^{2} \lambda_{E}^{2}(\mu)}{3}\left[1-\frac{\alpha_{s} C_{F}}{4 \pi}\left(2 L^{2}+2 L-\frac{2}{3}+\frac{5 \pi^{2}}{12}\right)\right. \\
\left.\left.+\frac{\alpha_{s} C_{G}}{4 \pi}\left(\frac{3}{4} L-\frac{1}{2}\right)\right]-\frac{t^{2} \lambda_{H}^{2}(\mu)}{6}\left[1-\frac{\alpha_{s} C_{F}}{4 \pi}\left(2 L^{2}+\frac{2}{3}+\frac{5 \pi^{2}}{12}\right)-\frac{\alpha_{s} C_{G}}{8 \pi}(L-1)\right]\right\}
\end{gathered}
$$

with the $\overline{\mathrm{MS}}$ scale $\mu$ and $\alpha_{s} \equiv \alpha_{s}(\mu)$, which takes into account the Wilson coefficients to $O\left(\alpha_{s}\right)$ and a complete set of the local operators of dimension less than 6 . Fourier transforming to the momentum representation and taking the first two regularized-moments, $\int_{0}^{\Lambda_{U V}} d \omega \omega^{j} \phi_{B}(\omega, \mu)(j=$ $0,1)$, the contributions from the first line in (4), associated with matrix element of the dimension3 and -4 operators, coincide completely with the result obtained in $[8]$. The second and third lines in (4) are generated from the dimension-5 operators. Our OPE result (4) "merges" the IR and UV structures peculiar to the $B$-meson LCWF [5, 6], and embodies novel behaviors that are completely different from those of the pion LCWF: $\mu$ and $t$ are strongly correlated due to the logarithmic contributions from cusp singularity in the radiative corrections, so that the WF is not Taylor expandable about $t=0$. The WF receives the contributions from many higher dimensional operators, in particular, from those associated with the long-distance gluon fields inside $B$-meson.

Our result (4) allows us to parameterize all nonperturbative contributions by a few HQET parameters. As a result, the $B$-meson LCWF (1) obeys the two-step evolution: it is governed by the Sudakov-type and Brodsky-Lepage-type scale dependence from the high scale $\mu_{i} \simeq \sqrt{m_{b} \Lambda_{\mathrm{QCD}}}$, associated with the QCD factorization formula, to the scale $\mu \lesssim 1 / t$, while that for the lower scale is governed by the anomalous dimensions of the local operators associated with the HQET parameters. This result is useful for clarifying model-independent properties of the $B$-meson LCWF, combined with nonperturbative estimates of the relevant HQET parameters.

K.T. thanks V. M. Braun for valuable discussions. This work is supported by the Grant-in-Aid for Scientific Research No. B-19340063.

\section{References}

[1] M. Beneke et al., Nucl. Phys. B591 (2000) 313; B606 (2001) 245; C. W. Bauer, D. Pirjol and I. W. Stewart, Phys. Rev. Lett. 87 (2001) 201806; H. n. Li, Prog. Part. Nucl. Phys. 51 (2003) 85. 
[2] A. G. Grozin and M. Neubert, Phys. Rev. D55 (1997) 272.

[3] M. Neubert, Phys. Rept. 245 (1994) 259.

[4] V. L. Chernyak and A. R. Zhitnitsky, Phys. Rept. 112 (1984) 173; V. M. Braun and I. E. Filyanov, Z. Phys. C48, 239 (1990); P. Ball, V. M. Braun, Y. Koike and K. Tanaka, Nucl. Phys. B529 (1998) 323.

[5] H. Kawamura, J. Kodaira, C.F. Qiao and K. Tanaka, Phys. Lett. B523 (2001) 111; Erratum-ibid. B536 (2002) 344; Mod. Phys. Lett. A18 (2003) 799; Nucl. Phys. B (Proc. Suppl.) 116 (2003) 269.

[6] B. O. Lange and M. Neubert, Phys. Rev. Lett. 91 (2003) 102001.

[7] V. M. Braun, D. Y. Ivanov and G. P. Korchemsky, Phys. Rev. D69 (2004) 034014.

[8] S. J. Lee and M. Neubert, Phys. Rev. D72 (2005) 094028.

[9] J. S. Schwinger, Phys. Rev. 82 (1951) 664; E. V. Shuryak and A. I. Vainshtein, Nucl. Phys. B199 (1982) 451; B201 (1982) 141; I. I. Balitsky and V. M. Braun, Nucl. Phys. B311 (1989) 541. 